\title{
The Use of Mechanical Testing, Photomicrography, and Electron Microscopy to Characterize an Insulating Fiberglass Composite Post-Electrical Arc Failure.
}

\author{
Robert J. Pieper ${ }^{1}$ \\ 1. Element Materials Technology, New Berlin, WI, USA
}

Thin multi-layered fiberglass composites composed of high temperature electrical grade epoxy with woven glass substrates are commonly used as insulating layers for electrical transformers and rotor generators. These engineered composites are widely used because of their excellent electrical properties and thermal stability, as well as their ability to retain flexibility under a wide range of conditions. During normal operating conditions the thin layered composites act as an insulating layer, having the ability to flex and still maintain structural integrity. However, once the ability for the composite to flex is removed, the fiberglass composite will typically crack leading to voids or air gaps within the layers. These voids will inevitably lead to an electrical discharge, compromising the composite layer and leading to electrical failure. Finding the cause of the electrical failure can sometimes be challenging, as the extreme heat and flame produced by the electrical arc will typically obliterate any evidence of the failure mode. The purpose of this study was to use photomicrography and electron microscopy to determine a potential mode of failure and simulate the mode of failure on new composite liners (lab sample) by mechanical compression testing according to ASTM D695. Once all testing was complete a comparison between the field failed sample and the lab failed sample was conducted.

The failed fiberglass composite liner was visually inspected and the hole in the liner from the arc was evident with the crack parallel to the length of the liner. On the bottom side of the liner was the residue from an adhesive used in the manufacturing process that ran parallel across the entire length. The presence of the adhesive resulted in only a small portion of the slot liner being mechanically secured, which allowed for the unsecured portion of the slot liner to flex at the point where the adhesive stopped. Any flexing could result in the formation of micro-fractures on the surface between the immovable/movable interfaces, which in turn can facilitate crack formation and extension.

The fracture and electrical arc sites labeled A, B, and C in Figure 1 was examined with the aid of a scanning electron microscope (SEM). Figure 2 shows locations A, B, and C, where individual fibers showed evidence of smooth fractured surfaces, which is a distinct characteristic of compressive force [1]. In addition, some individual fibers in location B showed two distinct areas of smooth compressive and rough tensile areas separated by a neutral axis, a typical feature of compressive failure. In the final fracture region, represented by Location $\mathrm{C}$, fiber fracture by micro-buckling is observed. This feature is characteristic of axial compression, and is typically revealed as stepped structures. Local fiber buckling and fiber fractures lead to an irregular, stepped fracture surface, which is a characteristic feature of compression failure [2]. Location $\mathrm{C}$ showed evidence of matrix and fiber debris, which are also characteristic of compression failed samples. Additional SEM images were taken of the lab samples after completion of the mechanical compression tests. The lab samples were compressed and the images were scanned from the top down on the failed specimen. Figure 3 shows the lab sample in increasing magnification. The scans clearly showed individual fibers with smooth fractured surfaces, similar to the failed sample depicted in Figure 2. The lab specimen had characteristics similar to the original failed sample, such as smooth fractured surfaces of the individual fibers and matrix/fiber debris scattered along the scanned area. Also similar is the lack of adhesion of the matrix/fibers. 
In conclusion, microscopic examination of the failed composite liner indicated potential failure via mechanical overload due to cyclic compression resulting in cracking from the area not mechanically adhered. To prove this, SEM images of the lab samples that were mechanically compressed to simulate compressive failure revealed similar fiber break features when compared to the failed samples. This indicates that one could simulate and characterize failure modes when the failed sample has limited evidence due post-failure destruction from heat, flame, or other contamination.

References:

[1] P. Stumpff, “Fractography,” in Composites, Volume 21, ASM International, 2001, Pages 977-987. [2] M. Ezrin, "Plastics Failure Guide, Cause and Prevention,” (Hanser/Gardner Publications, Inc, Cincinnati) p.326-331.

Figure 1. Locations chosen for SEM analysis.
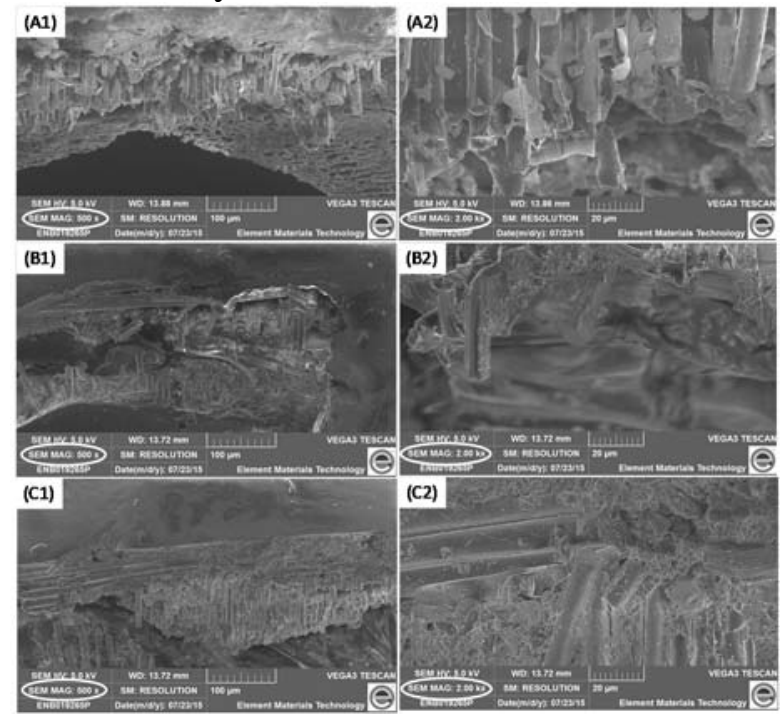

Figure 2. Locations A, B, and C shown in increasing magnification. Individual fibers show evidence of smooth compressive fractured surfaces, micro-buckling; with poor matrix/fiber adhesion.

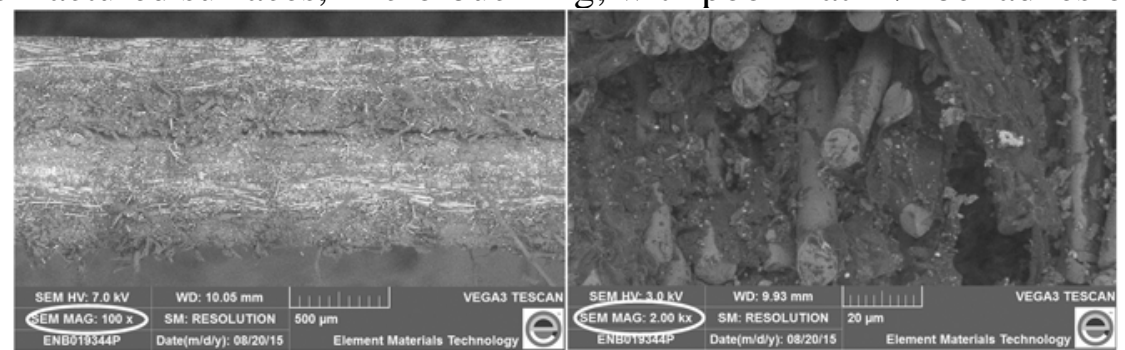

Figure 3. Lab simulated compression test shown in increasing magnification; with evidence of smooth surface fracture and good matrix/fiber adhesion. 